\title{
THREE DECADES OF ELECTRON BACKSCATTER DIFFRACTION OF MAGNESIUM: WHAT HAS IT TAUGHT US?
}

\author{
Matthew R. Barnett \\ Institute for Frontier Materials, Deakin University; Pigdons Rd; Geelong; 3217; Australia
}

\begin{abstract}
Electron backscatter diffraction has transformed microstructural analysis. Its advent enabled the microstructure to be correlated with crystallographic orientation in an unprecedented manner. This correlation has taught us many things about the physical metallurgy of magnesium and its alloys. Some highlights from three decades of research are presented in this talk. Chief among these is the insight the technique has provided into mechanical twinning, which plays a major role in the deformation of magnesium. The technique has also shed important light on deformation mechanisms, heterogeneity of material flow and, of course, the processes of texture development during wrought processing. It also promises more. In coming years, careful analysis of electron backscatter diffraction patterns and maps will in all likelihood continue to highlight the dominating mechanisms and phenomena that must be captured in microstructurally honest models of material response.
\end{abstract}

\section{Some Examples}

Electron backscatter diffraction (EBSD) capability became commercially available in 1986 [1]. Pettersen and Ryum [2] applied backscattered pattern analysis to a magnesium alloy in 1989. Since then many studies have applied the technique to this material, thereby shedding important new light on its physical metallurgy.

The technique involves automated indexing of Kikuchi diffraction patterns captured using a camera mounted behind a phosphor screen. Combined with beam rastering, this permits microstructure maps to be re-constructed from crystallographic orientation data. The technique has proven ideal for studying the occurrence of deformation twinning in magnesium and its alloys. Figure 1 provides an example of an image generated using the technique along with the secondary twins it was used to identify [3].

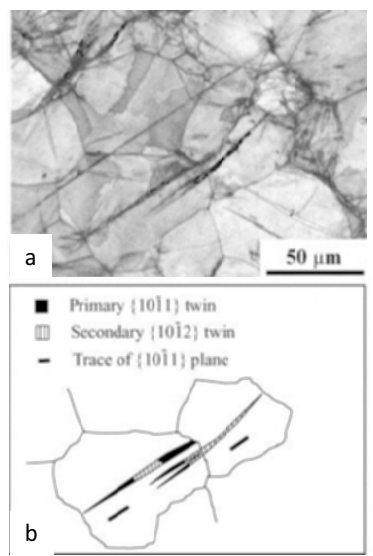

Figure 1. (a) EBSD map of pure Mg generated using Kikuchi pattern contrast, (b) identification of the double twins present [3].
The technique has been instrumental in verifying the prevalence of this secondary twinning reaction in commercial magnesium. EBSD also provided indication that such twinning events may be involved with void formation and ductile failure [4]. An example is provided in Figure 2. Although twin identification is difficult in this image, twins appear to be associated with the voids and fragments of double twin boundaries are able to be identified in twins parallel to the voids. Other notable studies that have exploited EBSD to shed important light on twinning in magnesium include those by the McGill [5] and Los Alamos [6] groups, for example.

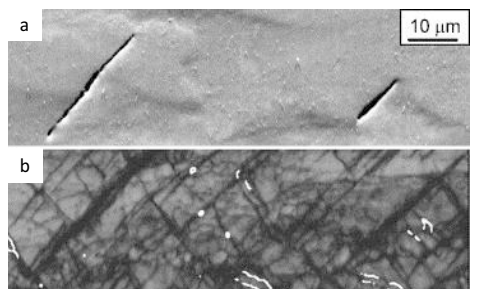

Figure 2. (a) Secondary electron image of voids in a sample of extruded ZK60 deformed to failure in tension, (b) fragments of double-twin boundaries highlighted in white [4].

The technique has also revealed the microstructural origin of the deformation texture of magnesium alloys containing rare-earth elements. One example is shown in Figure 3. Here, the inverse pole figure of the extrusion direction is shown for horizontally aligned grain clusters and for grain clusters formed in shear bands. It is seen that the two microstructural locations produce different orientations. Unique "rare-earth" textures can, in certain circumstances, be due to a predominance of the nucleation of recrystallization in shear bands [7].

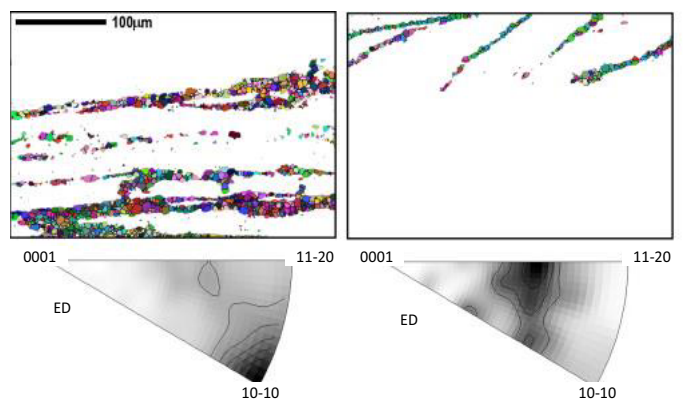

Figure 3. Crystallographic orientations produced from different microstructural regions of extruded alloy $\mathrm{Mg}-\mathrm{Gd}$. [7]

[1] Maitland, T and Sitzmanin, S. in Scanning Microscopy for Nanotechnology, Zhou, W, Wang Z.L. eds, Springer (2007), 41.

[2] Pettersen, K., Ryum, N. Metall. Trans. A, 20(1989) 847.

[3] Barnett, M.R., Nave, M.D., Bettles, C.J. Mat. Sci. Eng. A, (2004) 386, 205.

[4] Barnett, M.R., Mat. Sci. Eng. A (2007) 464, 8.

[5] Jiang, L., Jonas, et al. Scripta Mat. (2006) 54, 771.

[6] Beyerlein, I.J., Capolungo, et al. Phil. Mag. (2010) 90, 2161.

[7] Stanford, N., Barnett, M.R. Mat. Sci. Eng. A, (2008) 496, 399. 